\title{
Time Perspective and Bedtime Procrastination: The Role of the Chronotype-Time Perspective Relationship
}

\author{
Dexin Meng ${ }^{1, *}$ \\ Ying Zhao ${ }^{2,3, *}$ \\ Jing Guo ${ }^{2,3}$ \\ Huiying $X u\left(\mathbb{D}^{4}\right.$ \\ Yiming $\mathrm{Fu}^{2,3}$ \\ Xiaohan $\mathrm{Ma}^{2,3}$ \\ Liwei Zhu ${ }^{2,3}$ \\ $\mathrm{Li} \mathrm{Mu}{ }^{2,3}$
}

'Department of Physiology, Jiamusi University, Jiamusi, People's Republic of China; ${ }^{2}$ Research Center of Brain and Cognitive Neuroscience, Liaoning Normal University, Dalian, Liaoning Province, People's Republic of China; ${ }^{3}$ Key Laboratory of Brain and Cognitive Neuroscience, Liaoning Province, Dalian, Liaoning Province, People's Republic of China; ${ }^{4}$ School of Clinical Medicine, Changchun University of Chinese Medicine, Changchun, People's Republic of China

*These authors contributed equally to this work

\begin{abstract}
Introduction: Bedtime procrastination (BP) has a close relationship with one's chronotype, from a biological perspective on time. However, it remains unknown whether there is an association between BP and psychological time. Therefore, the aim of the present study was to evaluate the relationship between time perspective (TP) and BP and the effect of TP on the relationship between BP and chronotype by examining a sample of college students pre- and post-COVID-19 outbreak.
\end{abstract}

Methods: A total of 628 Chinese students (267 in pre-outbreak and 361 in post-outbreak) validly completed the Chinese version of the Bedtime Procrastination Scale, the Zimbardo Time Perspective Inventory (ZTPI), and the Morningness-Eveningness Questionnaire.

Results: Students with more BP behaviors exhibited greater deviation from a balanced TP, especially after the COVID-19 outbreak. Hierarchical regression analyses revealed that the pastnegative and future orientations, as operationalized by the ZTPI, contributed independently to BP behaviors. The structural equation modeling analyses further demonstrated that morningness was significantly related to a more future-oriented TP, which in turn decreased BP in pre- and postCOVID-19 outbreak samples, while morningness was associated with a less past-negativeoriented TP, which in turn decreased BP only in the post-COVID-19 outbreak sample.

Conclusion: This study indicated that TP in students with BP predominantly focused on future orientation and that TP can mediate the relationship between chronotype and BP behaviors. However, the COVID-19 pandemic may disrupt the time flow and change the role of chronotype-TP in BP. These findings explain how individual differences in TP are associated with BP, which may be helpful in designing effective interventions to avoid BP, from the viewpoint of time perspective therapy.

Keywords: bedtime procrastination, deviation from balanced time perspective, future time perspective, morningness, past negative time perspective, COVID-19

\section{Introduction}

Bedtime procrastination (BP) is a type of health behavior procrastination ${ }^{1}$ that has many negative consequences, such as sleep disturbances ${ }^{2,3}$ and mood disorders. ${ }^{4}$ Kroese et al defined BP as "failing to go to bed at the intended time while no external circumstances prevent a person from doing so."1 Some studies have indicated that BP is most likely to occur because of mindless delay, characterized by losing track of time due to immersive activities before sleeping (eg using electronic media). ${ }^{5}$ Therefore, BP may be associated with a variety of time-related variables.
Correspondence: $\mathrm{Li} \mathrm{Mu}$

Research Center of Brain and Cognitive Neuroscience, Liaoning Normal

University, No. 850 Huanghe Road,

Shahekou District, Dalian, II6029,

Liaoning Province, People's Republic of

China

Email mul@Innu.edu.cn 
A growing body of studies supports the assumption that time influences people's behaviors, and it has been recognized as an important factor generating individual differences in behaviors. ${ }^{6,7}$ From traditional biological approaches, chronotype is usually used to investigate the impact of time on behaviors. ${ }^{8}$ Prior studies have shown that evening-type persons ("owls") tend to report more BP behaviors than morning-type ones ("larks"), especially on working days, ${ }^{9,10}$ which is consistent with expectations of BP from a chronobiological perspective.

From a psychological perspective, however, it remains unknown whether there is an association between BP and time. Given its temporal nature, some studies have demonstrated that procrastination is linked to time perspective (TP) ${ }^{11}$ which is a conceptual model developed by Zimbardo and Boyd ${ }^{12}$ and refers to the individual temporal orientation related to a person's experiences and conceptions of the past, present, and future. Zimbardo's TP theory usually refines the three major time zones of the past, present, and future and disintegrates them into five temporal orientations: past-positive, past-negative, presenthedonistic, present-fatalistic, and future. ${ }^{12}$ The previous study has suggested that trait procrastination reflects a disjunction between the present and future with respect to cognitive temporal focus. ${ }^{11}$ Specifically, it is known to have a positive relationship with a present-hedonistic time orientation (an orientation toward present pleasure with little concern for future consequences) and a negative correlation with a future time orientation (a broad orientation toward the future goal-setting and forward-thinking, often at the expense of present enjoyment). ${ }^{11}$

In particular, in recent years, the concept of procrastination has been extended to the field of health behaviors. ${ }^{13}$ Studies have increasingly demonstrated that TP predicts a number of important health behaviors and outcomes, such as smoking cessation, dieting, and exercise. ${ }^{14,15}$ However, existing knowledge regarding the association between sleep outcomes and TP is very limited. The first direct evidence on sleep quality and TP was a short report based on a sample of students, and only a weak correlation was found. ${ }^{16}$ Recently, Ronnlund and Carelli proposed that a predominant past-negative orientation (an orientation toward the focus on negative, aversive experiences of the past) is positively associated with poor sleep quality, while future orientation is negatively related to poor sleep quality. ${ }^{6}$

Previous studies have revealed that people with greater BP behaviors were more likely to exhibit general procrastination and poor sleep quality. ${ }^{1,2}$ Thus, we hypothesized that TP could be associated with BP and might be an important factor causing individual differences in BP behavior. It is worth noting that researchers have previously revealed a close relationship between TP and chronotype ${ }^{17-19}$ Chronotype is significantly correlated with some dimensions in the theoretical framework of Zimbardo's TP, ${ }^{12}$ as well as with the Deviation from Balanced Time Perspective (DBTP). ${ }^{19}$ DBTP is an indicator that assesses the degree of deviation from a balanced TP, integrating different TPs into a balanced profile and emphasizing that individuals have the mental ability to switch flexibly between temporal orientations. ${ }^{20}$ It is commonly believed that larks are associated with being more future-oriented and owls with being more present- and/or past-oriented. ${ }^{17,19}$ However, no study has discussed the possible chronotype-TP relationship in BP behaviors. Therefore, we also hypothesized that TP might affect the relationship between chronotype and BP behaviors.

In addition, the coronavirus disease 2019 (COVID-19) pandemic has caused profound changes in health behaviors. ${ }^{21}$ Some studies have repeatedly reported that COVID-19-related sleep disruptions and changes in sleep patterns are common in the general population. ${ }^{22-24}$ For example, Lee et al found that during the COVID-19 pandemic quarantine, sleep onset on weekdays was delayed by nearly $30 \mathrm{~min}$ among Chinese people, ${ }^{22}$ suggesting that BP behaviors may be more evident than usual due to unprecedented restrictions during the COVID-19 pandemic.

In light of the description given above, the present study was aimed at evaluating the effects of TP on BP, as well as on the relationship between chronotype and BP behaviors. In particular, the study also attempted to compare the influence of the chronotype-TP relationship on BP pre- and post-COVID-19 outbreak. These results are expected to provide evidence for formulating BP interventions based on individuals' TPs.

\section{Materials and Methods}

\section{Participants and Procedure}

A total of 674 Chinese college students in public universities volunteered to participate in the survey. Nearly $40 \%$ of the respondents $(267 / 674)$ were recruited through online and offline advertisements in one university between April and December 2019 and received monetary compensation after completing the survey in the lab. The remaining $60 \%$ 
(407/674) were enrolled from the other two universities between May 11, 2020 and June 4, 2020, and were given no monetary compensation after completing the online survey at home. During this period, all schools in China were shut down, and students were quarantined to study at home to prevent the spread of COVID-19. No COVID-19 patients or close contacts were included in the postCOVID-19 outbreak sample.

In addition, all participants were engaged in non-shift work or study for the preceding one month, had not undertaken any cross-time-zone travel in the preceding three months and had no diagnosed sleep disorder. They were informed about all relevant aspects of the study and gave verbal or written informed consent. The informed consent process was approved by the Ethics Committee of Liaoning Normal University, and this study was conducted in accordance with the Declaration of Helsinki.

Participants needed to complete a series of web-based questionnaires about demographic characteristics (age, sex, and years of education), BP, TP and chronotype. Of the 674 students who responded, 46 were excluded due to their careless responses. The final statistical analysis included 628 valid respondents $(93.2 \%$ effective response rate).

\section{Measures}

\section{Bedtime Procrastination Scale (BPS)}

The Chinese version of the BPS is adapted from Kroese's English version of the BPS ${ }^{1}$ and has been used to assess BP behaviors among Chinese university students. ${ }^{2,4}$ The scale consists of nine items, each rated on a 5-point Likert scale ranging from 1 (never) to 5 (always). Among these, four items (Items 2, 3, 7, and 9) are reverse scored. The BPS score was computed by taking the average scores of the nine items, with a higher score indicating more BP behaviors. In the current study, the Cronbach's $\alpha$ for pre- and post-COVID19 outbreak samples were 0.867 and 0.833 , respectively.

\section{Morningness-Eveningness Questionnaire (MEQ)}

The MEQ was developed by Horne and Ostberg and consists of 19 items in a 4- or 5-Likert type response format to assess an individual's chronotype. ${ }^{25}$ The score is obtained by totalling the item scores, and ranges from 16 (extreme eveningness) to 86 (extreme morningness). The Chinese version of the MEQ has been revised by Zhang et al and is considered a reliable and valid measurement of circadian rhythm. ${ }^{26}$ The Cronbach's $\alpha$ for pre- and post-COVID-19 outbreak samples were 0.738 and 0.763 , respectively.

\section{Zimbardo Time Perspective Inventory (ZTPI)}

TP was measured using the validated Chinese version of the ZTPI ${ }^{27}$ which was originally developed by Zimbardo and Boyd, ${ }^{12}$ and has been used to assess TP in the Chinese population. ${ }^{28,29}$ This scale contains 56 items assessing five dimensions of TP: past-negative - a generally negative view of the past; past-positive - a warm and nostalgic attitude to past events; present-hedonistic - attraction toward immediate pleasure with little consideration of future consequences; present-fatalistic - a feeling of helplessness regarding the present, and considering the future as predestined; and future - a broad orientation toward the future. ${ }^{12}$ Each item is answered on a 5-point Likert scale, ranging from 1 (very uncharacteristic) to 5 (very characteristic). The Cronbach's $\alpha$ coefficients for the five dimensions ranged from 0.656 to 0.835 in the pre-COVID-19 outbreak sample and from 0.660 to 0.850 in the postCOVID-19 outbreak sample. A composite score for each subscale was computed by averaging the responses for each item, with higher scores reflecting stronger orientations towards a temporal frame.

Balanced TP integrates different TPs into an even profile, and was proposed to emphasize the importance of an individual's TP profile. ${ }^{20}$ The DBTP, as an indicator of the balanced TP, was calculated using a specific formula, to reflect individuals' ability to switch between TPs and to determine the extent to which scores deviated from zero (the balanced ideal). ${ }^{30}$ The optimal values for each dimension of ZTPI are: 1.95 for past-negative, 3.9 for present-hedonism, 4.0 for future, 4.6 for past-positive and 1.5 for present-fatalism. Higher DBTP scores indicate low levels of balanced TPs. Well-balanced individuals are those with a DBTP score $\geq 1 S D$ below the mean. ${ }^{31}$

\section{Statistical Analysis}

IBM SPSS Statistics version 21.0 for Windows and Amos version 24.0 (SPSS Inc., Chicago, IL, USA) was used for data processing. There were no missing values in the final statistical analyses. The distribution of each variable was determined by the one-sample Kolmogorov-Smirnov test. Data with normal distribution were expressed as mean and standard deviation (SD), while data with non-normal distribution were expressed as median and interquartile range. The categorical variables were expressed as frequency and proportion. Group comparisons in demographic characteristics, BP, MEQ, and ZTPI scores were evaluated with the Mann-Whitney $U$-test for continuous variables with non- 
normal distribution and Chi-square test for categorical variables. Pearson's (for variables with normal distribution) or Spearman's (for variables with non-normal distribution) correlation coefficients were calculated to assess the associations of BP with demographic characteristics, chronotype, and TP in pre- and post-COVID-19 outbreak groups, respectively. Bonferroni corrections were used to adjust for multiple corrections. Hierarchical regression analyses further investigated the effects of demographic characteristics, chronotype and TP on BP in pre- and postCOVID-19 outbreak groups, respectively. The BPS score was used as the dependent variable; demographic characteristics were used as covariates in step 1; the MEQ score was included in step 2; and the ZTPI dimensions were used as independent variables in step 3. Sex, age, and years of education were assessed as covariates owing to their significant correlation with BP, MEQ, and/or TP in the current correlation analyses, as well as in previous studies. $^{32-37}$ Variance inflation factor values exceeding 5 were used to indicate multicollinearity. To further explore the mediating role of TPs in the relationship between BP and chronotype, structural equation modeling (SEM) was established by Amos in pre- and post-COVID-19 outbreak groups, respectively. The independent variable was the
MEQ score, and the mediators were the dimensions of ZTPI, which might have statistical significance in hierarchical regression analyses. The BPS score was the dependent variable and demographic variables were entered as covariates. The model fit was judged using $\chi^{2} /$ df, root mean square error of approximation (RMSEA), goodness fit index (GFI), comparative fit index (CFI), normed fit index (NFI), Tucker-Lewis fit index (TLI) and incremental fit index (IFI). The statistical significance of path was evaluated using 5000 bootstrap resamples and bias-corrected and accelerated 95\% confidence intervals (BCa 95\% CI) excluding zero. Complete or partial mediation was assessed by testing whether the z-score was statistically significant (absolute value larger than 1.96). Parallel mediation analysis was used to evaluate which dimensions of ZTPI might show significant mediating effects. Differences were considered to be statistically significant at $p<0.05$.

\section{Results}

\section{Descriptive Statistics and Group Differences}

Table 1 presents the descriptive statistics of the demographic variables, BPS, MEQ, and ZTPI in the overall

Table I Differences in Demographic Characteristics, BP, Chronotype and TP Between Pre- and Post-COVID-19 Outbreak Groups

\begin{tabular}{|c|c|c|c|c|c|}
\hline Variables & All Sample & Pre-COVID-19 Outbreak & Post-COVID-19 Outbreak & $U I \chi^{2}$ & $p$ \\
\hline \multicolumn{6}{|l|}{ Demographic characteristics } \\
\hline $\mathrm{n}$ & 628 & 267 & 361 & & \\
\hline Sex & & & & 2.930 & 0.087 \\
\hline Male & $182(29.0)^{c}$ & $87(32.6)^{c}$ & $95(26.3)^{c}$ & & \\
\hline Female & $446(71.0)^{c}$ & $180(67.4)^{c}$ & $266(73.7)^{c}$ & & \\
\hline Age, years & $20.00(2.00)^{b}$ & $20.00(3.00)^{b}$ & $19(1.00)^{\mathrm{b}}$ & $34,105.000$ & $<0.001$ \\
\hline Education, years & $12.00(2.00)^{b}$ & $14.00(3.00)^{b}$ & $12(0.00)^{\mathrm{b}}$ & $14,289.000$ & $<0.001$ \\
\hline BPS & $2.89(1.00)^{b}$ & $2.76(0.83)^{\mathrm{a}}$ & $3.00(0.89)^{\mathrm{b}}$ & $56,776.500$ & $<0.001$ \\
\hline MEQ & $52.00(11.00)^{\mathrm{b}}$ & $51.84(8.39)^{\mathrm{a}}$ & $53.00(11.00)^{\mathrm{b}}$ & $48,831.500$ & 0.776 \\
\hline \multicolumn{6}{|l|}{ ZTPI } \\
\hline Past-negative & $3.15(0.90)^{\mathrm{b}}$ & $3.15(0.67)^{\mathrm{a}}$ & $3.10(0.80)^{\mathrm{b}}$ & $46,858.500$ & 0.552 \\
\hline Present-hedonistic & $3.13(0.53)^{b}$ & $3.25(0.47)^{\mathrm{a}}$ & $3.07(0.47)^{\mathrm{b}}$ & $38,557.500$ & $<0.001$ \\
\hline Future & $3.38(0.62)^{b}$ & $3.46(0.62)^{\mathrm{b}}$ & $3.38(0.54)^{b}$ & $40,257.000$ & $<0.001$ \\
\hline Past-positive & $3.56(0.67)^{\mathrm{b}}$ & $3.67(0.56)^{b}$ & $3.44(0.67)^{\mathrm{b}}$ & $36,375.000$ & $<0.001$ \\
\hline Present-fatalistic & $2.89(0.78)^{b}$ & $2.89(0.78)^{b}$ & $3.00(0.67)^{\mathrm{b}}$ & $49,925.000$ & 0.440 \\
\hline DBTP score & $2.54(0.76)^{\mathrm{b}}$ & $2.42(0.86)^{b}$ & $2.65(0.7 \mathrm{I})^{\mathrm{b}}$ & $56,694.000$ & $<0.001$ \\
\hline Low level of DBTP & $82(13.1)^{c}$ & $36(13.5)^{c}$ & $46(12.7)^{c}$ & 0.074 & 0.785 \\
\hline
\end{tabular}

Notes: ${ }^{a}$ Variables are normally distributed. Data are presented as mean and standard deviation. ${ }^{b}$ Variables are non-normally distributed. Data are presented as median and interquartile range. ${ }^{C}$ The categorical variables are presented as frequency and proportion. Statistically significant values $(p<0.05)$ were formatted in bold.

Abbreviations: BP, bedtime procrastination; BPS, Bedtime Procrastination Scale; COVID-19, Coronavirus Disease 20I9; DBTP, Deviation from Balanced Time Perspective; MEQ, Morningness-Eveningness Questionnaire; n, number; TP, time perspective; ZTPI, Zimbardo Time Perspective Inventory. 
sample. Almost $30 \%$ of the sample were males $(n=182)$ and $70 \%$ were females $(n=446)$. The age of participants ranged from 17 to 29 years (mean \pm SD: $20.20 \pm 1.75$ ) and years of education ranged from 12 to 19 years (mean \pm SD: $13.09 \pm 1.52$ ). Interestingly, participants, on average, reported moderate scores for BPS (mean \pm SD: $2.89 \pm$ 0.78 , ranging from 1 to 5 ) and the five ZTPI dimensions (mean from 2.87 to 3.53 , ranging from 1 to 5), suggesting that these phenomena are commonly experienced in this sample.

Table 1 also shows differences in demographic variables, BPS, MEQ, and ZTPI between pre- and post-COVID-19 outbreak samples. There were significant differences in age $(U=34,105.000, p<0.001)$ and years of education $(U=14,289.000, p<0.001)$ between pre- and post-COVID19 outbreak samples, except for sex $\left(\chi^{2}=2.930, p=0.087\right)$. Moreover, the BPS scores were significantly higher in the post-COVID-19 outbreak group than in the pre-COVID-19 outbreak group $(U=56,776.500, p<0.001)$. Nonetheless, students in the post-COVID-19 outbreak group had higher DBTP $(U=56,694.000, p<0.001)$ and lower ZTPI subscale scores of present-hedonistic $(U=38,557.500, p<0.001)$, future $(U=40,257.000, p<0.001)$, and past-positive $(U=$ $36,375.000, p<0.001)$ orientations, compared to the preCOVID-19 outbreak group. However, no significant differences in MEQ and ZTPI subscale scores of past-negative and present-fatalistic were found between pre- and post-COVID19 outbreak groups ( $p s>0.05$ ). Additionally, the proportion of participants with low levels of DBTP in pre-COVID-19 outbreak $(13.5 \%, \mathrm{n}=36)$ was similar to that in post-COVID-19 outbreak $(12.7 \%, \mathrm{n}=46)$, indicated by a DBTP score of $\leq$ (mean - 1SD)

\section{Correlations Between BP and TP}

Table 2 shows the correlations between BPS and demographic characteristics, ZTPI, and MEQ in pre- and postCOVID-19 outbreak groups, respectively. Age and years of education were negatively associated with the BPS score $\left(r_{\mathrm{age}}=-0.158, p=0.010\right.$ and $\left.r_{\text {education }}=-0.144, p=0.019\right)$, but positively correlated with the MEQ score $\left(r_{\text {age }}=0.182\right.$, $p=0.003$ and $\left.r_{\text {education }}=0.160, p=0.009\right)$, future-oriented $\operatorname{TP}\left(r_{\text {age }}=0.188, p=0.002\right.$ and $\left.r_{\text {education }}=0.173, p=0.005\right)$, and past-positive-oriented TP $\left(r_{\text {age }}=0.141, p=0.021\right.$ and $r_{\text {education }}=0.165, p=0.007$ ) only in the pre-COVID-19 outbreak group. No significant correlation was found between sex and BP and TPs in both groups. However, in the post-COVID-19 outbreak group, there was a significant correlation between the MEQ score and sex (female $=1$ and male $=0 ; r=-0.125, p=0.017$ ), suggesting that women show more "owl"-ish tendencies than men. Additionally, Table 2 shows that age was negatively associated with present-hedonistic-oriented TP $(r=-0.114, p=0.030)$ in the post-COVID-19 outbreak group.

For the ZTPI in both pre- and post-COVID-19 outbreak, the past-negative and present-fatalistic orientations were positively correlated with BPS scores (pre-outbreak: $r_{\text {past-negative }}=$ $0.291, p<0.001$ and $r_{\text {present-fatalistic }}=0.290, p<0.001$; postoutbreak: $r_{\text {past-negative }}=0.194, p<0.001$ and $r_{\text {present-fatalistic }}=$ $0.107, p=0.043$ ) and negatively associated with MEQ scores (pre-outbreak: $r_{\text {past-negative }}=-0.112, p=0.068$ and $r_{\text {present- }}$

Table 2 Correlations Between BP, Demographic Characteristics, Chronotype and TP in Pre- and Post-COVID-I9 Outbreak Groups, Respectively

\begin{tabular}{|c|c|c|c|c|c|c|c|c|c|c|c|c|}
\hline No & Variables & I & 2 & 3 & 4 & 5 & 6 & 7 & 8 & 9 & 10 & II \\
\hline 1 & $\operatorname{Sex}^{\mathrm{a}}$ & & $-0.165^{* *}$ & -0.046 & 0.093 & $-0.125^{*}$ & -0.018 & -0.056 & 0.049 & 0.088 & 0.014 & -0.031 \\
\hline 2 & Age & 0.029 & & $0.164^{* *}$ & -0.086 & -0.150 & -0.032 & $-0.114^{*}$ & 0.041 & 0.005 & -0.014 & 0.044 \\
\hline 3 & Education & 0.110 & $0.895 * * *$ & & -0.038 & 0.063 & 0.026 & 0.087 & -0.004 & -0.021 & 0.103 & 0.045 \\
\hline 4 & BPS & 0.098 & $-0.158 * *$ & $-0.144^{*}$ & & $-0.493 * * *$ & $0.194^{* * * *}$ & 0.035 & $-0.172^{* *}$ & -0.053 & $0.107^{*}$ & $0.204^{* * * *}$ \\
\hline 5 & MEQ & 0.024 & $0.182^{* *}$ & $0.160^{* *}$ & $-0.518^{* * * b}$ & & $-0.172^{* *}$ & -0.058 & $0.162 * *$ & 0.005 & $-0.154 * *$ & $-0.168 * *$ \\
\hline 6 & Past-negative & -0.038 & -0.050 & -0.064 & $0.291^{* * * b}$ & $-0.112^{* b}$ & & $0.425 * * *$ & 0.016 & -0.041 & $0.546 * * *$ & $0.57 I^{* * * *}$ \\
\hline 7 & Present-hedonistic & 0.055 & -0.092 & -0.094 & $0.158^{* * b}$ & $-0.116^{b}$ & $0.343^{* * * b}$ & & $0.142 * *$ & $0.253 * * *$ & $0.420 * * *$ & 0.017 \\
\hline 8 & Future & 0.035 & $0.188^{* *}$ & $0.173^{* *}$ & $-0.389 * * *$ & $0.307 * * *$ & -0.099 & $-0.149 *$ & & $0.466 * * *$ & $-0.140 * *$ & $-0.436 * *$ \\
\hline 9 & Past-positive & 0.085 & $0.141^{*}$ & $0.165^{* *}$ & $-0.158 * *$ & $0.148 *$ & $-0.215 * * *$ & $0.145^{*}$ & $0.264^{* * * *}$ & & -0.070 & $-0.589 * * *$ \\
\hline 10 & Present-fatalistic & -0.033 & -0.060 & -0.089 & $0.290 * * *$ & $-0.195^{* *}$ & $0.539 * * *$ & $0.455 * * *$ & $-0.256 * * *$ & $-0.150 *$ & & $0.623 * * *$ \\
\hline 11 & DBTP score & -0.097 & -0.086 & -0.105 & $0.323 * * *$ & $-0.199 * *$ & $0.78 I^{* * * *}$ & $0.178^{* *}$ & $-0.317 * * *$ & $-0.526 * * *$ & $0.727 * * *$ & \\
\hline
\end{tabular}

Notes: ${ }^{a} \mathrm{~A}$ dummy variable is used to recode sex, defined as I for female and 0 for male. ${ }^{\mathrm{b}}$ The correlations between two normally distributed continuous variables using Pearson's correlation coefficient. Pre-COVID-19 outbreak group below the diagonal; Post-COVID-19 outbreak group above the diagonal. Bolded correlations remain statistically significant after Bonferroni corrections. ${ }^{*} p<0.05$, $* * p<0.01$, $* * * p<0.001$.

Abbreviations: BP, bedtime procrastination; BPS, Bedtime Procrastination Scale; COVID-19, Coronavirus Disease 20I9; DBTP, Deviation from Balanced Time Perspective; MEQ, Morningness-Eveningness Questionnaire; TP, time perspective. 
fatalistic $=-0.195, p=0.001$; post-outbreak: $r_{\text {past-negative }}=$ $-0.172, p=0.001$ and $r_{\text {present-fatalistic }}=-0.154, p=0.003$ ), while the future orientation was negatively associated with BPS scores (pre-outbreak: $r_{\text {future }}=-0.389, p<0.001$; postoutbreak: $\left.r_{\text {future }}=-0.172, p=0.001\right)$ and positively correlated with MEQ scores (pre-outbreak: $r_{\text {future }}=0.307, p<0.001$; post-outbreak: $r_{\text {future }}=0.162, p=0.002$ ). However, the present-hedonistic and past-positive orientations were associated with BP only in the pre-COVID-19 outbreak group ( $r_{\text {present- }}$ hedonistic $=0.158, p=0.010$ and $r_{\text {past-positive }}=-0.158, p=$ 0.010). Notably, the correlation between BP and DBTP was also significantly positive in both groups $(r=0.323$ and $r=0.204$, both $p<0.001$ ), indicating that more BP behaviors are linked to high levels of non-balanced TP. In addition, the MEQ scores were negatively correlated with the BPS in both groups $(r=-0.518$ and $r=-0.493$, both $p<0.001)$.

\section{Independent Relationships Between BP and TP}

To further identify the extent to which TP bias allowed the prediction of BP independently of demographic factors, the hierarchical multiple regression analyses were conducted in both groups (Table 3). For BP, the demographic characteristics in step 1 explained $3.5 \%\left(\Delta F_{(3,263)}=3.167, p=0.025\right)$ and $1.3 \%$ of the variance $\left(\Delta F_{(3,357)}=1.524, p=0.208\right)$ in pre- and
post-COVID-19 outbreak groups, respectively. Adding chronotype in step 2 significantly improved the models and explained an additional $25.3 \%$ and $25.8 \%$ of the variance in BP (pre-outbreak: $\Delta F_{(1,262)}=26.503, p<0.001$; post-outbreak: $\left.\Delta F_{(1,356)}=33.080, p<0.001\right)$; hence, MEQ was a significant predictor (pre-outbreak: $\mathrm{B}_{\mathrm{MEQ}}=-0.050, p<$ 0.001; post-outbreak: $\left.\mathrm{B}_{\mathrm{MEQ}}=-0.043, p<0.001\right)$. Similarly, adding ZTPI scores in step 3 significantly increased the explained variance by $10.0 \%$ (pre-outbreak: $\Delta F_{(5,257)}=$ 18.073, $p<0.001$ ) and 2.4\% (post-outbreak: $\Delta F_{(5,351)}=$ $16.330, p<0.001$ ), leading to a total explained variance of $38.8 \%$ and $29.5 \%$ in both groups. Thus, two dimensions of ZTPI (pre-outbreak: $B_{\text {past-negative }}=0.255, p=0.001$ and $B_{\text {future }}$ $=-0.411, p<0.001$; post-outbreak: $\mathrm{B}_{\text {past-negative }}=0.168, p=$ 0.013 and $\mathrm{B}_{\text {future }}=-0.188, p=0.049$ ) were significant predictors of BP in step 3. When TPs were added to the models, the effect of chronotype declined, but remained significant (pre-outbreak: $\mathrm{B}_{\mathrm{MEQ}}=-0.041, p<0.001$; post-outbreak: $\left.\mathrm{B}_{\mathrm{MEQ}}=-0.040, p<0.001\right)$.

\section{The Chronotype-TP Relationship in BP Behaviors}

As the reduction in the effect of chronotype suggested possible mediation upon the introduction of TPs to the model, SEM analyses with maximum likelihood

Table 3 Hierarchical Regression Analyses Exploring the Effects of Chronotype and TP on BP in Pre- and Post-COVID-I9 Outbreak Groups, Respectively

\begin{tabular}{|c|c|c|c|c|c|c|c|c|c|c|}
\hline \multirow[t]{3}{*}{ Variables } & \multicolumn{10}{|c|}{ BPS } \\
\hline & \multicolumn{4}{|c|}{ Pre-COVID-19 Outbreak } & \multirow[t]{2}{*}{$\Delta \mathbf{R}^{2}$} & \multicolumn{4}{|c|}{ Post-COVID-I 9 Outbreak } & \multirow[t]{2}{*}{$\Delta \mathbf{R}^{2}$} \\
\hline & B & $\boldsymbol{\beta}$ & $t$ & $p$ & & B & $\boldsymbol{\beta}$ & $t$ & $p$ & \\
\hline Step I & & & & & 0.035 & & & & & 0.013 \\
\hline Sex ${ }^{a}$ & 0.206 & 0.117 & 1.876 & 0.062 & & 0.117 & 0.070 & 1.311 & 0.191 & \\
\hline Age & -0.023 & -0.062 & -0.487 & 0.627 & & -0.052 & -0.076 & -1.343 & 0.180 & \\
\hline Education & -0.046 & -0.095 & -0.740 & 0.460 & & -0.003 & -0.002 & -0.042 & 0.966 & \\
\hline Step 2 & & & & & 0.253 & & & & & 0.258 \\
\hline MEQ & -0.050 & -0.511 & -9.653 & $<0.001$ & & -0.043 & -0.513 & -11.231 & $<0.001$ & \\
\hline Step 3 & & & & & 0.100 & & & & & 0.024 \\
\hline Past-negative & 0.255 & 0.208 & 3.380 & 0.001 & & 0.168 & 0.152 & 2.486 & 0.013 & \\
\hline Present-hedonistic & -0.068 & -0.039 & -0.654 & 0.514 & & -0.125 & -0.081 & -1.361 & 0.174 & \\
\hline Future & $-0.4 I I$ & -0.223 & -4.020 & $<0.001$ & & -0.188 & -0.103 & -1.978 & 0.049 & \\
\hline Past-positive & 0.018 & 0.011 & 0.207 & 0.836 & & 0.072 & 0.055 & 0.996 & 0.320 & \\
\hline Present-fatalistic & 0.057 & 0.042 & 0.629 & 0.530 & & -0.005 & -0.004 & -0.064 & 0.949 & \\
\hline
\end{tabular}

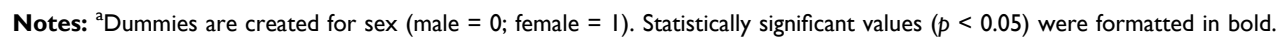

Abbreviations: BP, bedtime procrastination; BPS, Bedtime Procrastination Scale; COVID-19, Coronavirus Disease 20I9; MEQ, Morningness-Eveningness Questionnaire; TP, time perspective; B, Unstandardized regression coefficient; $\beta$, Standardized regression coefficient. 
estimation based on 5000 bootstrapped samples were performed in pre- and post-COVID-19 outbreak groups, respectively. The mediation model had an ideal fit to the data in the pre-COVID-19 outbreak group: $\chi^{2} / \mathrm{df}=1.743$, $p=0.065, \mathrm{RMSEA}=0.053, \mathrm{GFI}=0.982, \mathrm{CFI}=0.987$, NFI $=0.970$, TLI $=0.972$, IFI $=0.987$ (Figure 1$)$. The analysis confirmed a significant partial indirect effect of chronotype on BP through future orientation in the ZTPI
$\left(\mathrm{B}_{\text {future }}=-0.007, \mathrm{BCa} 95 \% \mathrm{CI}\right.$ from -0.124 to $-0.004, p<$ 0.001 ), accounting for nearly $20 \%$ of the mediating effect (Table 4). However, the indirect effect of past-negativeoriented TP was not significant in the pre-COVID-19 outbreak group $\left(\mathrm{B}_{\text {past-negative }}=-0.002, \mathrm{BCa} 95 \% \mathrm{CI}\right.$ from -0.006 to $0.000, p=0.072$ ).

The mediation model had an ideal fit to the data in the post-COVID-19 outbreak group: $\chi^{2} / \mathrm{df}=1.113, p=0.347$,

\section{Pre-COVID-19 outbreak}

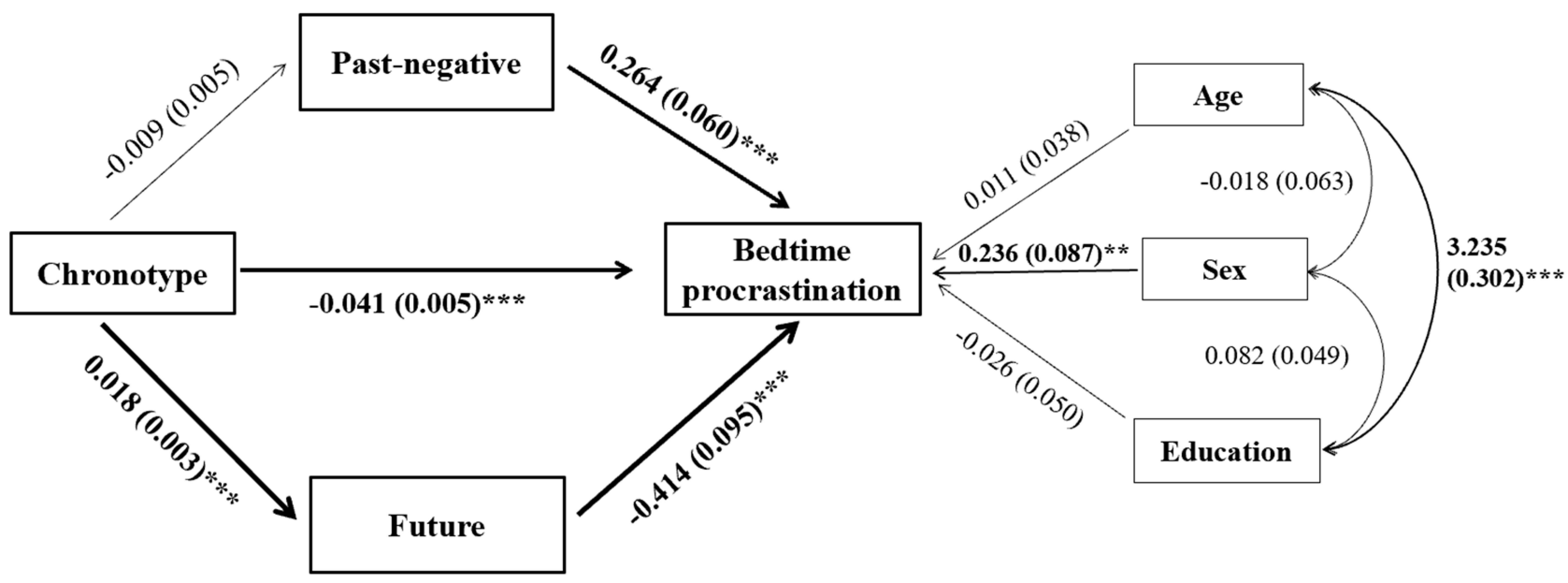

\section{Post-COVID-19 outbreak}

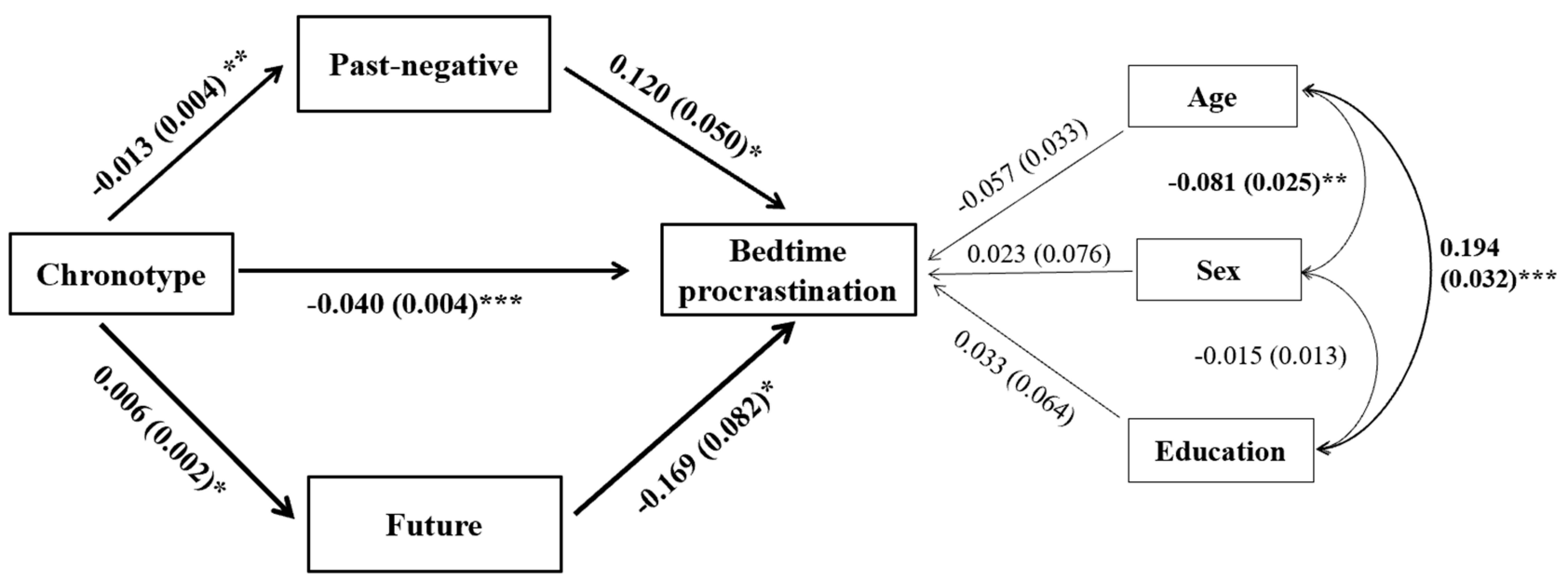

Figure I The results of structural equation modeling of the mediating roles of TPs in the association between BP and chronotype in pre- and post-COVID-19 outbreak samples. The effect of demographic characteristics (sex, age, and years of education) on BP was controlled in these models. All the coefficients in the figure are unstandardized. The model fit indices in the pre-COVID-19 outbreak sample: $\chi^{2} / \mathrm{df}=1.743, p=0.065, \mathrm{RMSEA}=0.053, \mathrm{GFI}=0.982, \mathrm{CFI}=0.987, \mathrm{NFI}=0.970, \mathrm{TLI}=$ 0.972 , IFI $=0.987$. The model fit indices in the post-COVID- 19 outbreak sample: $\chi^{2} / \mathrm{df}=1 . \mathrm{II}, p=0.347, \mathrm{RMSEA}=0.0 \mathrm{I} 8, \mathrm{GFI}=0.99 \mathrm{I}, \mathrm{CFI}=0.994, \mathrm{NFI}=0.946, \mathrm{TLI}=$ 0.987, IFI $=0.994 . * p<0.05, * * p<0.01, * * * p<0.001$.

Abbreviations: BP, bedtime procrastination; COVID-19, Coronavirus Disease 2019; TP, time perspective. 
Table 4 Unstandardized Direct, Indirect and Total Effects of SEM Models in Pre- and Post-COVID-19 Outbreak Groups, Respectively

\begin{tabular}{|c|c|c|c|c|c|c|c|}
\hline \multirow[t]{2}{*}{ Effects } & \multirow[t]{2}{*}{ Paths } & \multirow[t]{2}{*}{ Point Estimate } & \multicolumn{2}{|c|}{ Product of Coefficients } & \multicolumn{2}{|c|}{$\mathrm{BaC} \mathrm{95 \%} \mathrm{Cl}$} & \multirow[t]{2}{*}{$p$} \\
\hline & & & SE & $\mathbf{Z}$ & Lower & Upper & \\
\hline \multicolumn{8}{|c|}{ Pre-COVID-19 outbreak } \\
\hline Total effect & $\begin{array}{l}\text { Chronotype } \\
\downarrow\end{array}$ & -0.051 & 0.005 & -10.20 & -0.061 & -0.041 & $<0.001$ \\
\hline Indirect effect & $\begin{array}{l}\text { Future } \\
\downarrow\end{array}$ & -0.010 & 0.003 & -3.33 & -0.016 & -0.005 & $<0.001$ \\
\hline Direct effect & $\mathrm{BP}$ & -0.041 & 0.005 & -8.20 & -0.052 & -0.031 & $<0.001$ \\
\hline \multicolumn{8}{|c|}{ Post-COVID-19 outbreak } \\
\hline Total effect & $\begin{array}{l}\text { Chronotype } \\
\downarrow\end{array}$ & -0.043 & 0.004 & -10.75 & -0.051 & -0.035 & $<0.001$ \\
\hline Indirect effects & $\begin{array}{l}\text { Past-negative } \\
\text { Future } \\
\downarrow\end{array}$ & -0.003 & 0.001 & -3.00 & -0.005 & -0.001 & 0.010 \\
\hline Direct effect & $\mathrm{BP}$ & -0.040 & 0.004 & -10.00 & -0.048 & -0.032 & $<0.001$ \\
\hline
\end{tabular}

Notes: Unstandardized estimating of 5000 bootstrap sample. Statistically significant values $(p<0.05)$ were formatted in bold. $Z=$ Point Estimate/SE. If the absolute value of the $\mathrm{z}$-score is greater than 1.96 , the effect is considered significant.

Abbreviations: $\mathrm{BaC} 95 \% \mathrm{Cl}$, bias-corrected and accelerated $95 \%$ confidence intervals; BP, bedtime procrastination; COVID-I9, Coronavirus Disease 20I9; SE, standard error; SEM, structural equation modeling.

RMSEA $=0.018, \mathrm{GFI}=0.991, \mathrm{CFI}=0.994, \mathrm{NFI}=0.946$, TLI $=0.987$, IFI $=0.994$ (Figure 1$)$. The total effect of chronotype on BP was significant $\left(\mathrm{B}_{\text {total }}=-0.043, \mathrm{SE}=\right.$ $0.004, p<0.001)$, the direct effect was significant $\left(\mathrm{B}_{\text {direct }}=\right.$ $-0.040, \mathrm{SE}=0.004, p<0.001)$ and partial indirect effects are present $\left(\mathrm{B}_{\text {indirect }}=-0.003, \mathrm{SE}=0.001, p=0.010\right)$ (Table 4). Parallel mediation analysis confirmed that two mediators were significantly contributed to the overall indirect effects, accounting for nearly $7 \%$ of the mediating effect (Figure 1). Specifically, there was as statistically significant indirect effect of chronotype to BP though future- $\left(\mathrm{B}_{\text {future }}=-0.001, \mathrm{BCa} 95 \% \mathrm{CI}\right.$ from -0.003 to $-0.000, p=0.046)$ and past-negative-oriented TP $\left(\mathrm{B}_{\text {past- }}\right.$ negative $=-0.002, \mathrm{BCa} 95 \% \mathrm{CI}$ from -0.004 to $-0.000, p=$ 0.038 ), such that greater morningness was significantly related to a more future and a less past-negative orientations, which in turn decreased BP.

\section{Discussion}

This is the first study we know exploring the relationship between TP and BP in Chinese college students. In the beginning, our focus was on the balanced TP profiles obtained from the DBTP scores, which were based on the ZTPI subscale scores. The mean scores for each of the five ZTPI dimensions in this study differed from previously reported optimal values for $\mathrm{DBTP}^{38}$ and from the normative sample means reported by Zimbardo and Boyd. ${ }^{20}$ In the current study, DBTP scores were significantly higher in the post-outbreak group than in the pre-outbreak group, indicating a more significant DBTP after the COVID-19 outbreak. This is consistent with the view that the COVID-19 pandemic may distort the flow of time perception. ${ }^{39}$

However, there was no significant difference in the proportion of well-balanced students with a low level of DBTP pre- and post-COVID-19 outbreak of $13.5 \%$ and $12.7 \%$, respectively, which corresponds with the finding of a previous study using the same method (14.2\%, 18/ 127), ${ }^{31}$ but is significantly different from other studies that reported this as $23 \%$ using a hierarchical cluster analysis $^{40}$ and $5 \%(13 / 260)$ using the cut-off-point method. ${ }^{41}$ Differences in methods for measuring balanced TP may be responsible for this discrepancy. Furthermore, Zhang et al have found that the DBTP is the most appropriate method to measure balanced TP compared to the calculation methods mentioned above and can be used to accurately predict subjective well-being. ${ }^{42}$

Further, we found that the degree of non-balanced TP identified by the DBTP was positively related to BP among students in pre- and post-COVID-19 outbreak groups, suggesting that changes in TP may have 
significant implications for healthy behaviors. Our study also indicated that all ZTPI dimensions were associated with BP in the pre-COVID-19 outbreak group. Specifically, students with higher scores on the past-negative, present-hedonistic, and present-fatalistic subscales were more likely to report BP behaviors, contrary to participants with higher scores on the future and pastpositive subscales. At the same time, our results found that after the outbreak of COVID-19, students' past-positive and present-hedonism TPs rated lower and their BP behaviors increased significantly. Previous studies have shown that individuals with high-rating past-positive and present-hedonism orientations tend to have more life satisfaction, $^{43}$ and the COVID-19 pandemic has led to lower life satisfaction. ${ }^{44}$ However, present-hedonistic and past-positive time orientations were no longer associated with BP in the post-COVID-19 outbreak group, suggesting that the weak association between BP and these time orientations that positively affects well-being may exacerbate BP behaviors. Additionally, it indicates that the influence of changes of TP on BP may be mainly reflected in other time orientations during the COVID-19 pandemic.

Moreover, we examined which dimensions of ZTPI caused TP bias that independently influenced BP. The regression analysis demonstrated that the TP dimensions capturing past-negative and future orientations were independently related to BP behaviors in both pre- and postCOVID-19 outbreak samples. Our findings are consistent with previous studies on sleep, which have shown poor sleep quality to be positively associated with a past-negative TP and negatively associated with a future TP. ${ }^{6,45}$ As a sleep-related procrastination, BP typically leads to poor sleep quality; ${ }^{2}$ therefore, such consistent results are reasonable.

Interestingly, our hierarchical regression analyses demonstrated that chronotype accounted for a stable additional $25 \%$ of the variance in BP pre- and post-COVID-19 outbreak; whereas, TPs only accounted for an additional $2.4 \%$ of the variance in BP beyond chronotype in the post-COVID-19 outbreak group, which was lower than $10.0 \%$ in the pre-COVID-19 outbreak group. As a chronic and collective trauma, the COVID-19 pandemic has filled people with profound uncertainty and fear about the future. ${ }^{39}$ Future TP is defined as an individual's futureoriented cognitive, affective, and behavioral tendencies. Existing research states that it has a positive impact on many areas within people's lives, such as their tendency to take fewer health risks. ${ }^{46}$ Our data also showed a low future TP in the post-COVID outbreak sample. Thus, these results suggested that disrupting the time flow can significantly affect the consequences of healthy behaviors.

It is noteworthy that future orientation appears to be a prominent predictor of BP among all ZTPI dimensions, especially pre-COVID-19 outbreak, providing another example of future orientation's critical role in accounting for health-related behaviors. Our study also supports previous research that examined the effect of future TP on perceptions of health status and concluded that there was a significant association between future orientation and perceptions of good health. ${ }^{47}$

Furthermore, our results also support previous observations that morningness is associated with being more future-oriented $^{17,19}$ or less prone to $\mathrm{BP}, 9,10$ and eveningness with being more present- and past-oriented ${ }^{17,19}$ or more prone to BP. ${ }^{9,10}$ Altogether, we speculate that TP may have an indirect effect on the relationship between BP and the circadian rhythm (chronotype).

Not surprisingly, the results of our subsequent SEM analyses confirmed this assumption. The analyses demonstrated that greater morningness was significantly related to a more future-oriented TP, which in turn reduced the tendency to delay bedtime pre- and post-COVID-19 outbreak. The findings support and partly account for the link between chronotype and BP. Some studies have shown that social jetlag, a misalignment between the biological and social clocks, was considered an important precipitating factor of BP to explain the relationship between chronotype and BP. ${ }^{9}$ For example, Kuhnel et al found that morning types tended to have less social jetlag and reported less BP than the evening types. ${ }^{9}$ Moreover, the results of Borisenkov et al also showed that people with a future TP are less likely to have social jetlag. ${ }^{30}$ Thus, one plausible explanation is that chronic dysfunction of the circadian rhythm (social jetlag) can change long-term planning, which can be assessed by ZTPI, and especially change future TP in ZTPI, ${ }^{30}$ while people with future TP tend to display the characteristics of a slow life history strategy, ${ }^{48}$ which is characterized by less risky behavior and increased attention to their own health. ${ }^{49}$

In addition, past-negative $\mathrm{TP}$ is a negative, aversive, and ruminative orientation towards the past. Existing research has shown that owls were more past-oriented and larks were less past-oriented. ${ }^{32}$ Our findings also support this view. Further, the current SEM analysis found a partial indirect effect of past-negative TP between 
chronotype and BP in the post-COVID-19 outbreak sample. Some studies have shown that a high orientation towards the negative past is associated with increased stress and tension, especially in individuals with posttraumatic stress symptoms ${ }^{50}$ which may increase the risk of unhealthy behaviors. ${ }^{45,51}$ Indeed, in the context of trauma exposure caused by the COVID-19 pandemic, past-negative-oriented people have a pessimistic view of their lives and the world. ${ }^{39}$ Thus, our results linked such constructs characterized by negative affectivity of past time to BP and chronotype, demonstrating that greater morningness was significantly related to a less past-negative-oriented TP, which in turn reduced the tendency to delay bedtime post-COVID-19 outbreak.

Our study had several limitations. First, self-report data on BP are subjective, and more objective tools from sleep diaries or actigraphs need to be developed in the future. Second, existing studies have found that many of the original ZTPI dimensions are confounded by positive or negative valences ${ }^{17,52,53}$ and noted that the emotional valence of items on the ZTPI might affect the relationships between chronotype and TPs. ${ }^{17}$ Unfortunately, however, the current study did not exclude highly emotional ZTPI items to overcome the impact of valence on associations between BP, chronotype, and TP. Third, the MEQ, which was originally developed by Horne and Ostberg, ${ }^{25}$ only considers one dimension of the chronotype, that is, the phase of sleepwake cycle. However, it cannot be used to assess other main parameters describing circadian rhythm, such as the acrophase, the amplitude, and morning affect/alertness, which have been detected by several multidimensional assessment tools. ${ }^{54,55}$ Therefore, unfortunately, the current study cannot suggest whether the observed results might vary for amplitude of diurnal variation (distinctness), and morning affect/alertness, irrespective of morningness-eveningness preference. Fourth, associations between individual chronotype and certain personality traits or negative affect have been well established from previous studies. ${ }^{56,57}$ Likewise, increasing evidence also implies that chronotype is closely related to sleep quality and social jetlag. ${ }^{58}$ However, we did not collect the corresponding information in the present study. Fifth, only college students were selected for this study, thus the sample's generalizability was limited. Finally, we failed to take selfregulation into account. Indeed, a solid body of research has demonstrated significant associations between TP, chronotype and self-regulation. ${ }^{59,60}$ Given evidence that BP is robustly associated with chronotype and selfregulation, ${ }^{3,9,10,61}$ it appears reasonable for future studies to consider self-regulation in understanding the link between TP and BP.

\section{Conclusion}

In sum, this study demonstrated that DBTP-particularly, past-negative and future orientations - was independently related to more BP behaviors as operationalized by the ZTPI, in Chinese college students pre- and post-COVID19 outbreak. Furthermore, future orientation in ZTPI mediated the relationship between BP and chronotype pre- and post-COVID-19 outbreak. However, past-negative TP mediated the relationship between BP and chronotype only post-COVID-19 outbreak. These changes in perceptions of time may have significant implications for people's health and well-being, especially during the COVID-19 pandemic. In addition, Zimbardo and Boyd have suggested that TPs are malleable ${ }^{20}$ and put forward one new therapeutic approach, time perspective therapy, in order to be effectively correct and treat health damaging behaviors and diseases with temporal bias. ${ }^{62,63}$ Therefore, based on these findings of the relationship between TP and $\mathrm{BP}$, which explain how individual differences in TP are associated with BP, interventions with the potential to foster a more balanced TP may reduce the occurrence of $\mathrm{BP}$, and thus must be further explored.

\section{Abbreviations}

BP, bedtime procrastination; BPS, Bedtime Procrastination Scale; COVID-19, Coronavirus Disease 2019; DBTP, Deviation from Balanced Time Perspective; MEQ, Morningness-Eveningness Questionnaire; SEM, structural equation modeling; TP, time perspective; ZTPI, Zimbardo Time Perspective Inventory.

\section{Funding}

This work was supported by the National Natural Science Foundation of China (No.31800924) and the Scientific Study Project for Education Department of Liaoning Province (No.L201783641).

\section{Disclosure}

Dexin Meng and Ying Zhao are first authors. The authors declare no competing interests. 


\section{References}

1. Kroese FM, de Ridder DTD, Evers C, Adriaanse MA. Bedtime procrastination: introducing a new area of procrastination. Front Psychol. 2014;5:611. doi:10.3389/fpsyg.2014.00611.

2. Ma XH, Meng DX, Zhu LW, et al. Bedtime procrastination predicts the prevalence and severity of poor sleep quality of Chinese undergraduate students. J Am Coll Health. 2020:1-8. doi:10.1080/ 07448481.2020 .1785474$.

3. Kroese FM, Evers C, Adriaanse MA, de Ridder DTD. Bedtime procrastination: a self-regulation perspective on sleep insufficiency in the general population. J Health Psychol. 2016;21(5):853-862. doi:10.1177/1359105314540014.

4. Guo J, Meng DX, Ma XH, Zhu LW, Yang LM, Mu L. The impact of bedtime procrastination on depression symptoms in Chinese medical students. Sleep Breath. 2020;24(3):1247-1255. doi:10.1007/s11325020-02079-0.

5. Nauts S, Kamphorst BA, Stut W, de Ridder DTD, Anderson JH. The explanations people give for going to bed late: a qualitative study of the varieties of bedtime procrastination. Behav Sleep Med. 2019;17 (6):753-762. doi:10.1080/15402002.2018.1491850.

6. Ronnlund M, Carelli MG. Time perspective biases are associated with poor sleep quality, daytime sleepiness, and lower levels of subjective well-being among older adults. Front Psychol. 2018;9:1356. doi:10.3389/fpsyg.2018.01356.

7. Vitale JA, Weydahl A. Chronotype, physical activity, and sport performance: a systematic review. Sports Med. 2017;47(9):1859-1868. doi:10.1007/s40279-017-0741-z

8. Owens JA, Dearth-Wesley T, Lewin D, Gioia G, Whitaker RC. Selfregulation and sleep duration, sleepiness, and chronotype in adolescents. Pediatrics. 2016;138(6):e20161406. doi:10.1542/ peds.2016-1406.

9. Kuhnel J, Syrek CJ, Dreher A. Why don't you go to bed on time? A daily diary study on the relationships between chronotype, selfcontrol resources and the phenomenon of bedtime procrastination. Front Psychol. 2018;9:77. doi:10.3389/fpsyg.2018.00077.

10. Kadzikowska-Wrzosek R. Insufficient sleep among adolescents: the role of bedtime procrastination, chronotype and autonomous vs. controlled motivational regulations. Curr Psychol. 2020;39 (3):1031-1040. doi:10.1007/s12144-018-9825-7.

11. Sirois FM. Out of sight, out of time? A meta-analytic investigation of procrastination and time perspective. Eur J Pers. 2014;28 (5):511-520. doi:10.1002/per.1947.

12. Zimbardo PG, Boyd JN. Putting time in perspective: a valid, reliable individual-differences metric. J Pers Soc Psychol. 1999;77 (6):1271-1288. doi:10.1037/0022-3514.77.6.1271.

13. Kroese FM, de Ridder DTD. Health behaviour procrastination: a novel reasoned route towards self-regulatory failure. Health Psychol Rev. 2016;10(3):313-325. doi:10.1080/ 17437199.2015.1116019.

14. Hall PA, Fong GT, Meng G. Time perspective as a determinant of smoking cessation in four countries: direct and mediated effects from the International Tobacco Control (ITC) 4-country surveys. Addict Behav. 2014;39(7):1183-1190. doi:10.1016/j.addbeh.2014.03.019.

15. Guthrie LC, Butler SC, Lessl K, Ochi O, Ward MM. Time perspective and exercise, obesity, and smoking: moderation of associations by age. Am J Health Promot. 2014;29(1):9-16. doi:10.4278/ ajhp.130122-QUAN-39.

16. Vranesh JG, Madrid G, Bautista J, Ching P, Hicks RA. Time perspective and sleep problems. Percept Mot Skills. 1999;88(1):23-24. doi:10.2466/pms.1999.88.1.23.

17. Nowack K, van der Meer E. Are larks future-oriented and owls present-oriented? Age- and sex-related shifts in chronotype-time perspective associations. Chronobiol Int. 2013;30(10):1240-1250. doi:10.3109/07420528.2013.815197.
18. Milfont TL, Schwarzenthal M. Explaining why larks are future-oriented and owls are present-oriented: self-control mediates the chronotype-time perspective relationships. Chronobiol Int. 2014;31(4):581-588. doi:10.3109/07420528.2013.876428.

19. Stolarski M, Ledzińska M, Matthews G. Morning is tomorrow, evening is today: relationships between chronotype and time perspective. Biol Rhythm Res. 2013;44(2):181-196. doi:10.1080/09291016.20 12.656248

20. Zimbardo PG, Boyd JN. The Time Paradox: The New Psychology of Time That Will Change Your Life. New York (NY): Simon and Schuster; 2008.

21. Wang XQ, Lei SM, Le SL, et al. Bidirectional influence of the COVID-19 pandemic lockdowns on health behaviors and quality of life among Chinese adults. Int J Environ Res Public Health. 2020;17 (15):5575. doi:10.3390/ijerph17155575.

22. Lee PH, Marek J, Nálevka P. Crowdsourced smartphone data reveal altered sleep/wake pattern in quarantined Chinese during the COVID-19 outbreak. Chronobiol Int. 2020;37(8):1181-1190. doi:10.1080/07420528.2020.1792486

23. Martínez-de-quel Ó, Suárez-Iglesias D, López-Flores M, Pérez CA. Physical activity, dietary habits and sleep quality before and during COVID-19 lockdown: a longitudinal study. Appetite. 2021;158:105019. doi:10.1016/j.appet.2020.105019.

24. Shillington KJ, Vanderloo LM, Burke SM, Ng V, Tucker P, Irwin JD. Not so sweet dreams: adults' quantity, quality, and disruptions of sleep during the initial stages of the COVID-19 pandemic. Sleep Med. 2021. doi:10.1016/j.sleep.2021.02.028.

25. Horne JA, Ostberg O. A self-assessment questionnaire to determine morningness-eveningness in human circadian rhythms. Int J Chronobiol. 1976;4(2):97-110.

26. Zhang B, Hao YL, Rong RG. The reliability and validity of Chinese version morningness-eveningness questionnaire. Chin J Behav Med Sci. 2006;15(9):856-858. doi:10.3760/cma.j.issn.16746554.2006.09.044

27. Sircova A, van de Vijver FJR, Osin E, et al. A global look at time: a 24-country study of the equivalence of the zimbardo time perspective inventory. SAGE Open. 2014;4(1):1-12. doi:10.1177/2158244 013515686.

28. Ge JJ, Wu J, Li KS, Zheng Y. Self-compassion and subjective well-being mediate the impact of mindfulness on balanced time perspective in Chinese college students. Front Psychol. 2019;10:367. doi:10.3389/fpsyg.2019.00367.

29. Unger A, Yan J, Busch R. The relationship between the zimbardo time perspective inventory and violations of business ethics in China: unbalanced time perspective increases the acceptance of unethical business behavior. Time Soc. 2016;28(1):83-106. doi:10.1177/ $0961463 \times 16634723$.

30. Borisenkov MF, Vetosheva VI, Kuznetsova YS, et al. Chronotype, social jetlag, and time perspective. Chronobiol Int. 2019;36 (12):1772-1781. doi:10.1080/07420528.2019.1683858.

31. Mooney A, Earl JK, Mooney CH, Bateman H. Using balanced time perspective to explain well-being and planning in retirement. Front Psychol. 2017;8:1781. doi:10.3389/fpsyg.2017.01781.

32. Randler C, Engelke J. Gender differences in chronotype diminish with age: a meta-analysis based on morningness/chronotype questionnaires. Chronobiol Int. 2019;36(7):888-905. doi:10.1080/ 07420528.2019 .1585867$.

33. Zhu LW, Meng DX, Ma XH, Guo J, Mu L. Sleep timing and hygiene practices of high bedtime procrastinators: a direct observational study. Fam Pract. 2020;37(6):779-784. doi:10.1093/fampra/ cmaa079.

34. Zerbini G, Merrow M. Time to learn: how chronotype impacts education. Psych J. 2017;6(4):263-276. doi:10.1002/pchj.178.

35. Siu NYF, Lam HHY, Le JJY, Przepiorka AM. Time perception and time perspective differences between adolescents and adults. Acta Psychol. 2014;151:222-229. doi:10.1016/j.actpsy.2014.06.013. 
36. Andre L, van Vianen AEM, Peetsma TTD, Oort FJ. Motivational power of future time perspective: meta-analyses in education, work, and health. PLoS One. 2018;13(1):e0190492. doi:10.1371/journal. pone. 0190492 .

37. Schuster M, Oberlinner C, Claus M. Shift-specific associations between age, chronotype and sleep duration. Chronobiol Int. 2019;36(6):784-795. doi:10.1080/07420528.2019.1586719.

38. Stolarski M, Wiberg B, Osin E. Assessing temporal harmony: the issue of a balanced time perspective. In: Stolarski M, Fieulaine N, van Beek W, editors. Time Perspective Theory; Review, Research and Application. 1 ed. New York (NY): Springer, Cham; 2015:57-71.

39. Holman EA, Grisham EL. When time falls apart: the public health implications of distorted time perception in the age of COVID-19. Psychol Trauma. 2020;12:S63-S65. doi:10.1037/tra0000756.

40. Boniwell I, Osin E, Linley PA, Ivanchenko GV. A question of balance: time perspective and well-being in British and Russian samples. $J$ Posit Psychol. 2010;5(1):24-40. doi:10.1080/ 17439760903271181.

41. Drake L, Duncan E, Sutherland F, Abernethy C, Henry C. Time perspective and correlates of well-being. Time Soc. 2008;17 (1):47-61. doi:10.1177/0961463X07086304.

42. Zhang JW, Howell RT, Stolarski M. Comparing three methods to measure a balanced time perspective: the relationship between a balanced time perspective and subjective well-being. $J$ Happiness Stud. 2013;14(1):169-184. doi:10.1007/s10902-012-9322-x.

43. Zhang JW, Howell RT. Do time perspectives predict unique variance in life satisfaction beyond personality traits? Pers Individ Dif. 2011;50(8):1261-1266. doi:10.1016/j.paid.2011.02.021.

44. Ammar A, Chtourou H, Boukhris O, et al. COVID-19 home confinement negatively impacts social participation and life satisfaction: a worldwide multicenter study. Int J Environ Res Public Health. 2020;17:17. doi:10.3390/ijerph17176237.

45. Rönnlund M, Åström E, Westlin W, et al. A time to sleep well and be contented: time perspective, sleep quality, and life satisfaction. Front Psychol. 2021;12:627836. doi:10.3389/fpsyg.2021.627836.

46. Abousselam N, Naudé L, Lens W, Esterhuyse K. The relationship between future time perspective, self-efficacy and risky sexual behaviour in the black youth of central South Africa. J Ment Health. 2016;25(2):176-183. doi:10.3109/09638237.2015.1078884.

47. Kooij DTAM, Kanfer R, Betts M, Rudolph CW. Future time perspective: a systematic review and meta-analysis. J Appl Psychol. 2018;103(8):867-893. doi:10.1037/ap10000306.

48. Stolarski M, Czarna AZ, Malesza M, Szymańska A. Here and now: sociosexuality mediates the associations between dark triad and time perspectives (in females). Pers Individ Dif. 2017;111:119-123. doi:10.1016/j.paid.2017.02.001.

49. Ellis BJ, Figueredo AJ, Brumbach BH, Schlomer GL. Fundamental dimensions of environmental risk: the impact of harsh versus unpredictable environments on the evolution and development of life history strategies. Hum Nat. 2009;20(2):204-268. doi:10.1007/ s12110-009-9063-7.

50. Walg M, Eder LL, Martin A, Hapfelmeier G. Distorted time perspective in adolescent Afghan and Syrian refugees is associated with psychological distress. J Nerv Ment Dis. 2020;208(9):729-735. doi:10.1097/nmd.0000000000001194.
51. Chavarria J, Allan NP, Moltisanti A, Taylor J. The effects of present hedonistic time perspective and past negative time perspective on substance use consequences. Drug Alcohol Depend. 2015;152:39-46. doi:10.1016/j.drugalcdep.2015.04.027.

52. McGowan NM, Brannigan R, Doyle D, Coogan AN. Diurnal preference, circadian phase of entrainment and time perspectives: just what are the relationships? Pers Individ Dif. 2017;112:79-84. doi:10.1016/j.paid.2017.02.051.

53. Nowack K, Milfont TL, van der Meer E. Future versus present: time perspective and pupillary response in a relatedness judgment task investigating temporal event knowledge. Int $J$ Psychophysiol. 2013;87(2):173-182. doi:10.1016/j.ijpsycho.2012.12.006.

54. Randler C, Díaz-Morales JF, Rahafar A, Vollmer C. Morningnesseveningness and amplitude - development and validation of an improved composite scale to measure circadian preference and stability (MESSi). Chronobiol Int. 2016;33(7):832-848. doi:10.3109/ 07420528.2016.1171233.

55. Dosseville F, Laborde S, Lericollais R. Validation of a chronotype questionnaire including an amplitude dimension. Chronobiol Int. 2013;30(5):639-648. doi:10.3109/07420528.2012.763042.

56. Stolarski M, Gorgol J, Matthews G. The search for causality of personality-chronotype associations: insights from a one-year longitudinal study of adolescents. Chronobiol Int. 2021;38(4):489-500. doi: $10.1080 / 07420528.2020 .1867157$.

57. Cox RC, Olatunji BO. Differential associations between chronotype, anxiety, and negative affect: a structural equation modeling approach. J Affect Disord. 2019;257:321-330. doi:10.1016/j.jad.2019.07.012.

58. Raman S, Coogan AN. A cross-sectional study of the associations between chronotype, social jetlag and subjective sleep quality in healthy adults. Clocks Sleep. 2020;2(1):1-6. doi:10.3390/ clockssleep2010001.

59. Baird HM, Webb TL, Martin J, Sirois FM. The relationship between time perspective and self-regulatory processes, abilities and outcomes: a protocol for a meta-analytical review. BMJ Open. 2017;7 (6):e017000. doi:10.1136/bmjopen-2017-017000.

60. Kim J, Hong H, Lee J, Hyun MH. Effects of time perspective and self-control on procrastination and internet addiction. J Behav Addict. 2017;6(2):229-236. doi:10.1556/2006.6.2017.017.

61. Kamphorst BA, Nauts S, de Ridder DTD, Anderson JH. Too depleted to turn in: the relevance of end-of-the-day resource depletion for reducing bedtime procrastination. Front Psychol. 2018;9:252. doi:10.3389/fpsyg.2018.00252.

62. Sword RM, Sword RKM, Brunskill SR. Time perspective therapy: transforming zimbardo's temporal theory into clinical practice. In: Stolarski M, Fieulaine N, van Beek W, editors. Time Perspective Theory; Review, Research and Application. 1 ed. New York (NY): Springer, Cham; 2015:481-498.

63. Hall PA, Fong GT. The effects of a brief time perspective intervention for increasing physical activity among young adults. Psychol Health. 2003;18(6):685-706. doi:10.1080/0887044031000110447.
Nature and Science of Sleep

\section{Publish your work in this journal}

Nature and Science of Sleep is an international, peer-reviewed, open access journal covering all aspects of sleep science and sleep medicine, including the neurophysiology and functions of sleep, the genetics of sleep, sleep and society, biological rhythms, dreaming, sleep disorders and therapy, and strategies to optimize healthy sleep.
The manuscript management system is completely online and includes a very quick and fair peer-review system, which is all easy to use. Visit http://www.dovepress.com/testimonials.php to read real quotes from published authors. 